\title{
Derechos colectivos y Teoría del Derecho. Más ideología que teoría Collective Rights and Legal Theory. More ideology than theory
}

\author{
José María Sauca \\ Universidad Carlos III de Madrid \\ ORCID ID 0000-0001-5472-4491 \\ josemaria.sauca@uc3m.es
}

Cita recomendada:

Sauca, J.M. (2019). Derechos colectivos y teoría del Derecho. Más ideología que teoría. Eunomía. Revista en Cultura de la Legalidad, 17, 100-115.

doi: https://doi.org/10.20318/eunomia.2019.4993

Recibido / received: 28/08/2019

Aceptado / accepted: 21/09/2019

\begin{abstract}
Resumen
El tratamiento teórico de los derechos colectivos y su carácter polémico mantienen una importante actualidad tanto desde la perspectiva filosófico política y moral como desde la correspondiente a la dogmática jurídica. Sin embargo, los tratamientos de dicha categoría formulados por la teoría jurídica están recibiendo un menor e injustificado interés. En el presente trabajo se presta especial atención al estudio de los trabajos de Caracciolo y Jovanović en la materia y se concluye en que las pretendidas aproximaciones teóricas de análisis conceptual colapsan en una aproximación ideológica sobre la aceptación o rechazo de la categoría.
\end{abstract}

\section{Palabras clave}

Teoría e ideología, derechos, derechos culturales.

\section{Abstract}

The theoretical treatment of collective rights and their controversial nature maintain important relevance both from the political and moral philosophical perspective and from that corresponding to legal dogmatics. However, the treatments of this category formulated by legal theory are receiving less and unjustified attention. In this paper, special attention is given to the study of Caracciolo, and Jovanovic's respective works on the subject. It concludes that the intended theoretical approaches of conceptual analysis collapse into an ideological approach on the acceptance or rejection of the category.

\section{Keywords}

Theory and ideology, rights, cultural rights.

Sumario. 1. Actualización del panorama teórico sobre la temática de los derechos colectivos. 2. Propuestas críticas sobre la admisibilidad de la categoría de derechos colectivos de Ricardo Caracciolo. 3. Propuestas de justificación de los derechos colectivos sobre el valor del colectivismo de Miodrag A. Jovanović. 4. Consideraciones finales. 


\section{Actualización del panorama teórico sobre la temática de los derechos colectivos}

Parece un lugar común señalar que la temática de los derechos colectivos ha ido permanentemente de la mano con el desarrollo de los derechos individuales, aunque nunca ha alcanzado un estatuto de consolidación mínimamente equiparable. A pesar de ello, las tres últimas décadas han visto florecer una rica literatura académica sobre la materia y parece que su vigor dista de haberse agotado. Una aleatoria búsqueda en la Red, mediante un programa al uso en contextos académicos, arroja más de tres millones de entradas sobre trabajos académicos en la temática tan solo en inglés y español ${ }^{1}$. Esta exuberancia bibliográfica, cuya extensión me anima a no anegar este trabajo en la ímproba labor de dar cuenta de la misma, se reparte, de manera poco sorpresiva, entre las reflexiones sobre la fundamentación de los derechos colectivos (y de sus críticos) y los estudios dogmáticos sobre sus concreciones normativas. Los primeros son desarrollados por filósofos políticos acompañados, en ocasiones, de filósofos morales, mientras que los segundos se reparten entre internacionalistas, por un lado, y constitucionalistas, por otro, frecuentemente acompañados de laboralistas especializados en la negociación colectiva y administrativistas de perfil urbanístico y medioambientalista.

Entre ambos grandes sectores se abre paso, sin embargo, un tipo de reflexión mucho más restringida y que se corresponde con la autoría de los filósofos del Derecho. El tipo de argumentación seguido por estos suele resultar deudora de los dos estilos generales. Así, por un lado, se da una mayoritaria línea de trabajo que recoge la reflexión filosófico política y la acerca a la configuración de la teoría jurídica de los derechos fundamentales. Por otro, suele partir de los textos normativos que recogen dimensiones de titularidad de derechos atribuidos a colectivos y tratar de operar un proceso de abstracción tendente a la configuración de una justificación de la categoría en cuestión. Como ejemplo de la primera estrategia significaría la selección de trabajos publicados en el monográfico publicado en el número 2 Canadian Journal of Law and Jurisprudence de 1991 y que constituye una piedra de toque del moderno debate en la materia. En la literatura local destacaría los trabajos de García Inda (2001), Torbisco (2006), Sauca (2010 y 2014) y Bengoetxa (2015), así como los fructíferos análisis críticos de Rodríguez Abascal (2002 y 2003) y González Amuchastegui (2004). Como ejemplo de la segunda, destacaría los esfuerzos de MacCormick (1977 y 1982) y de Kramer (2002 y 2017). Ambos desarrollan actualizaciones -por cierto, incompatibles entre sí- de las teorías del interés como soporte de los derechos subjetivos en clave de revisión de su categorización hohfeldiana, que les lleva a establecer las bases para una teoría de los derechos colectivos fundamentada en el interés propio del grupo en el mantenimiento del colectivo. Recojo una conclusión clara del segundo:

Solo en la medida de que los miembros son constitutivos de un grupo, tienen, sin embargo, un interés directo en la cohesión del grupo. Qua asociación, los miembros tienen colectivamente un interés directo en estar juntos (como una asociación); después de todo, salvo que se mantengan juntos perderán su propia continuidad como asociación. Este interés directo de los miembros qua colectividad no puede ser reducido a una diversidad de intereses indirectos, en parte, porque ninguna diversidad de intereses indirectos puede, por ellos mismos, conformar en total un interés directo $\mathrm{y}$, en parte, porque el interés directo puede permanecer incluso cuando los intereses indirectos se cambian o parcialmente desaparecen (Kramer, 2002, p. 55 [las cursivas son del autor]).

\footnotetext{
${ }^{1}$ Me refiero al uso de Google Académico que cuantifica 2.800.000 entradas para trabajos académicos ante la entrada de collective rights y de 692.000 para la de derechos colectivos. Los cálculos se disparan hasta trescientos veintidós millones para la primera y cuarenta y siete millones para la segunda cuando abrimos la consulta en la red sin filtros académicos (u.c.21/07/2019).
} 
Entre nosotros merecen una especial mención los trabajos de López Calera (1992 y 2000) que han tenido una amplia influencia. En su estrategia, la constatación de la existencia de una multiplicidad de entidades jurídicas de carácter colectivo y titulares de derechos constituye la base normativa insoslayable de que existen los derechos colectivos y de que la reflexión iusfilosófica debe dar cuenta de la justificación de dichas entidades jurídicas. En último término, como decía Kelsen, el Derecho es como un Rey Midas que convierte en Derecho todo lo que toca (2008, p. 57). Si el Derecho crea entes colectivos y les atribuye derechos, solo desde presupuestos externos a una teoría del Derecho se puede continuar alegando su inexistencia.

Sin embargo, los filósofos del Derecho que han intentado una aproximación genuinamente conceptual son mucho menos numerosos. En este esfuerzo, considero que merecen un reconocimiento expreso los casos de $\operatorname{Raz}(1986,1994$ y 1998) y su propuesta de justificación de los derechos colectivos como derechos a bienes colectivos y de Réaume y su propuesta de justificación de los derechos colectivos como derechos a bienes participativos. En la academia hispanohablante, creo que quien ha intentado seguir esta senda es Cruz Parcero (1998 y 2007) quien, recogiendo expresamente la clasificación de Alexy sobre aproximaciones analíticas a la categoría de derechos, ha ido evolucionando en favor de un mayor margen de aceptación y pertinencia de la misma.

En este contexto, estimo, sin embargo, que hay dos autores encuadrados en la adscripción disciplinar de la teoría jurídica que han recibido una atención mucho menor de la que se les debe prestar y que esta deficiencia debe ser corregida. Me refiero a los trabajos de, por un lado, Ricardo Caracciolo y, por otro, de Miodrag A. Jovanović. En estas páginas prestaré atención al análisis que cada una de las propuestas, crítica la primera y justificadora la segunda, prestan al análisis teórico jurídico de los derechos colectivos.

2. Propuestas críticas sobre la admisibilidad de la categoría de derechos colectivos de Ricardo Caracciolo

La temática de los derechos colectivos se presenta como un aspecto colateral y singular de la obra de Caracciolo. El trabajo fundamental en que aborda la cuestión se titula ¿Existen los derechos colectivos? y fue publicado inicialmente en 2002, en un libro editado a cargo de Susanna Pozzolo (Caracciolo, 2002). Posteriormente, ha sido incluido como capítulo de cierre en el libro que se publicó bajo el título de El Derecho desde la Filosofía. Ensayos (Caracciolo, 2009). Es precisamente en términos de ensayo el modo en que Caracciolo se acerca a esta cuestión que, en lo que se me alcanza, resulta inédita en el conjunto de su obra. Destaco su carácter ensayístico tanto por razones metodológicas como sustantivas. En el primer sentido, Caracciolo soslaya una aproximación sistemática a la cuestión y, a lo largo de los siete puntos que conforman su cuerpo, va delimitando una estrategia expositiva que transita desde el cuestionamiento existencial de la categoría al problema metaético subyacente a la fundamentación de un eventual tipo peculiar de los mismos que se englobaría bajo la idea de derechos a la identidad cultural. En el segundo sentido, probablemente este artículo sea el trabajo en que Caracciolo se acerca más y más abiertamente a temas colindantes con la filosofía política y aunque mantiene intacto su estilo argumentativo, incluido el recurso a la formalización de las tesis en examen, el razonamiento llega a adquirir un claro sentido normativo. Con todo, creo importante significar que el carácter fronterizo que esta temática tiene para Caracciolo no se presenta, a mi modo de ver, como una curiosidad o incluso una relativa extravagancia, sino que implica la selección de un tema en el que ensayar la intersección de tres de las líneas más 
potentes de su pensamiento. Me refiero al concepto de norma, al de racionalidad colectiva y al de evaluación moral del Derecho².

Desde estos parámetros, paso a exponer cuáles son sus tesis centrales, con las que adelanto parciales discrepancias que intentaré ir explicando simultáneamente.

El punto de partida de Caracciolo es clásico en el autor: la intensa controversia filosófica sobre los derechos colectivos radica en confusiones conceptuales de diversa índole por lo que una labor de depuración conceptual resulta básica. «En rigor -afirma- se trata de saber exactamente qué se pretende decir cuando se lo afirma [los derechos colectivos] o cuando se lo niega» (2009, p. 261). A tal fin propone una estrategia definicional mixta a caballo entre estipulativa y lexicográfica: «tendré que formular -advierte- algunas estipulaciones, que se justifican (en parte) por la utilización de esa expresión en la literatura corriente» (2009, p. 261).

Las estipulaciones son tres:

En primer lugar, hay una contraposición entre derechos individuales y derechos colectivos. Esta contraposición radica en que los derechos colectivos tienen como objeto un bien colectivo y los derechos individuales tienen como contenido acciones $u$ omisiones o sus resultados.

En segundo lugar, un bien colectivo es un bien que pertenece a un grupo y no a los individuos que lo integran.

En tercer lugar, los derechos sobre bienes colectivos sólo pueden ser derechos de un grupo, esto es, derechos colectivos.

Las estipulaciones como tales no detentan valor de veracidad o falsedad, pero si cabe evaluar la mayor o menor utilidad de las mismas. En este sentido, creo que podrían apuntarse algunas sugerencias en relación con las ideas expuestas.

Con respecto a la primera, tendría que señalar que se me escapa la utilidad de introducir un desequilibrio categorial entre el contenido de los derechos colectivos y el de los individuales o, al menos, uno como el que se propone. Dos serían los tipos de argumentos. Por un lado, el objeto o contenido sobre el que versa un derecho es un bien, cualesquiera sean las condiciones relativas de su carácter público o privado y ello no es privativo de los derechos colectivos. Por decirlo con un ejemplo, tanto es un bien el bien colectivo de disfrutar de una ciudad limpia como el bien particular de tener limpio mi jardín. Por otro lado, los derechos, en cuanto atribución de facultades, implican acciones, omisiones o sus resultados tanto en el caso de que aquellas estén atribuidas a individuos como a colectividades. En general, la producción de bienes colectivos amerita una mayor cantidad de acciones y omisiones que un derecho individual. El hecho de que suela ser así no desdibuja la característica de que exige, precisamente, acciones y omisiones o sus resultados.

En relación con la segunda, concuerdo en que hay dificultades para delimitar las características de un bien como colectivo, aunque en la mayoría de los casos la idea gira en torno al carácter inexcuible de su disfrute, al menos, mediante procedimientos que no atenten a los derechos individuales del excluido de su disfrute. Sin embargo, tal y como está formulada, la tesis de que un bien colectivo es un bien que pertenece a un grupo y no a los individuos que lo integran es ambigua. Si por pertenencia a un grupo entendemos la titularidad del grupo, esta interpretación colapsa con la tercera tesis, por lo que resulta redundante. La alternativa supondría distinguir dos sentidos en la vinculación entre bien colectivo y grupo. Por un lado, el que se refiere a la producción del bien y, por otro, al disfrute del mismo. En el primero

\footnotetext{
2 Estos son algunos de los temas centrales del autor. Pienso en alguno de sus trabajos clásicos como La noción de sistema en la teoría del Derecho (1994) «Realismo moral vs. positivismo jurídico» (2001), «Derecho y decisiones colectivas» (1991) o «Acción colectiva y justicia» (2006).
} 
de ellos, concordaría sobre la base de que, generalmente, la producción de un bien colectivo requiere la participación de un número importante de individuos o en que, necesariamente, es así cuando el bien colectivo tiene carácter participativo. En el segundo, sin embargo, no. Un individuo, en tanto que individuo, puede tener acceso al disfrute de un bien colectivo. Por ejemplo, una campaña pública de vacunación contra enfermedades contagiosas de un grupo con especial riesgo a sufrirlas -el bien colectivo de la salud del grupo- concurre con el derecho individual a la protección de su salud como disfrute de una protección individual.

En cuanto a la tercera tesis -los derechos sobre bienes colectivos sólo pueden ser derechos de un grupo, esto es, derechos colectivos-, estimo que debería recibir alguna matización. Por un lado, cabe la posibilidad de mantener que algunos derechos sobre bienes colectivos pueden ser individuales -en línea de Raz y en contra de MacCormick (Rèaume, 1998, p. 9)-como, por ejemplo, el derecho individual de un niño a ser escolarizado en una determinada lengua como consecuencia de su inclusión en un grupo al que se reconoce tal derecho de protección lingüística o el derecho de un indígena a la explotación de los recursos naturales o de la pesca porque pertenece a un pueblo indígena al que se reconoce la titularidad de esos bienes. De últimas, tiene razón Buchanan cuando distingue tres tipos de ejercicio de un derecho colectivo: conjuntamente, por representante o individualmente (Buchanan, 1994 , p. 12). Por otro lado, no hay una continuidad necesaria entre derechos de grupo y derechos colectivos. Los primeros harían referencia a derechos de grupo diferenciado, que implican una situación de desequilibrio frente a grupos culturales mayoritarios, mientras que los segundos harían referencia a derechos a cualesquiera bienes colectivos (Kymlicka, 1995).

A partir de estas estipulaciones, Caracciolo restringe el objeto de análisis a los derechos colectivos relativos a la preservación o conservación de un rasgo cultural identificador de un grupo. Dos consideraciones acompañan esta opción. Por un lado, las conclusiones de este debate pueden ser generalizadas. Por otro, son los que comportan las controversias más intensas en la discusión teorética contemporánea. Asimismo, y a mi modo de ver, de manera promisoria, evita anegar el discurso en los debates relativos a la plausibilidad de la tesis sobre la relevancia que para la identidad personal de cualquier individuo presenta su pertenencia a un grupo. En otras palabras, los criterios por los que se define la identidad de un grupo de relevancia identitaria, así como cuáles de sus rasgos culturales le confieren identidad. Sin embargo, como diré más adelante, su discurso vuelve sobre estas cuestiones.

De esta forma, Caracciolo parte de un supongamos; la hipótesis es que un cierto grupo $\mathrm{G} 1$ tiene el derecho colectivo $\mathrm{D}$ al rasgo cultural identificatorio R1. Sobre la base de la tercera estipulación antes señalada, todos los enunciados que atribuyan un derecho individual con dichas características son falsos o carecen de sentido. Pero sobre qué bases, pregunta Caracciolo, se pueden mantener estos derechos. La dicotomía que presenta es la siguiente: o bien se sostiene una (extraña) ontología sobre los rasgos culturales o bien se interpretan como prácticas colectivas que resultan ser agregados de acciones individuales. Rechazada la primera alternativa como inviable (solo algunas tesis holísticas fuertes, como las del pionero en la materia Van Dyke, recurrirían a ella ${ }^{3}$ ), la segunda se presenta como la única opción aceptable. Los derechos colectivos a la identidad cultural serían $x 1 D / p_{2}$ siendo " $p$ " las prácticas en cuestión. Esta opción implica algún tipo de reduccionismo de los derechos colectivos en derechos individuales. Este reduccionismo puede ser fuerte o débil. El primero supondría afirmar que los enunciados existenciales de los derechos colectivos son directamente equivalentes a aquellos que afirman la existencia de los

\footnotetext{
${ }^{3}$ Por todos, véase Vernon Van Dyke (1974, pp. 725-741; 1980, pp. 1- 21).
} 
derechos individuales. El segundo supondría afirmar que existen derechos colectivos si, y sólo si, existen los correspondientes derechos individuales.

El primero es rechazable porque la existencia de derechos individuales no implica la existencia de un grupo, ni de un rasgo cultural determinado a proteger, ni del derecho de una entidad colectiva a detentar un derecho. Tomados desde el punto de vista de los presuntos derechos colectivos, éstos no serían más que un recurso retórico para reforzar los derechos individuales o algunos derechos individuales.

El segundo, sostenible para los críticos de los derechos colectivos (Barry, 2001, p. 112) y posiblemente para el propio Caracciolo, presupone la idea de la fundamentación de un derecho en la existencia de un interés suficientemente relevante como para justificar la imposición de deberes a otros. Desde esta idea, negar el reduccionismo débil supone afirmar que hay intereses de grupos distintos a los individuos que los componen y eventualmente incompatibles con los intereses de éstos últimos. Afirmar esta última tesis, concluye Caracciolo, no se justifica y duda de que pueda ser sostenida por alguna posición.

Creo que la justificación del profesor argentino para sostener el reduccionismo débil debiera ser revisada porque resulta ambigua. Concuerdo en que no es adecuado plantear la existencia de entes colectivos diferentes a los que se componen de individuos y que pudieran tener un tipo de existencia autónoma y diferenciada de éstos. Sin embargo, hay una versión menor o más débil de esta misma tesis que consiste en no negar que dichos grupos puedan responder a una estructura más compleja que la mera agregación de los individuos que lo componen. Como dice expresamente el último Raz: «el interés de grupo no puede ser reducido a interés individual» (Margalit y Raz, 1995, p. 87). Estimo que puede plantearse que la negación de la existencia de grupos distintos al simple sumatorio de los individuos que los componen supone una reductibilidad del grupo en sus componentes que no está acreditada. Creo que aquí Caracciolo incurre, por un lado, en una confusión entre explicabilidad y reductibilidad en la relación entre grupo y los individuos que lo conforman y, por otro, desestima la singularidad de los derechos que protegen prácticas colectivas en tanto que son prácticas participativas.

Para exponer la primera idea recurro al ejemplo que propone Kramer para explicar la confusión que le imputa a Hohfeld cuando éste descalifica la posibilidad de entender que un grupo puede ser algo diferente a sus miembros.

Supongamos -dice Kramer- que alguien declara que un cierto grupo de gente es grande. Obviamente, tal declaración no sostiene que los miembros de ese grupo son grandes; pueden ser enanos. Pero la declaración describe una característica del grupo que puede ser perfectamente explicada por referencia a sus miembros individuales. Más exactamente, la extensión de un grupo puede ser explicada por referencia a la multiplicidad de sus miembros. Una vez que los miembros de un grupo han pasado cierto nivel -un nivel que será definido de manera diferente por diversos observadores- el grupo será calificado como grande. Ninguna otra cosa más que el número de los miembros cuenta para el tamaño del grupo (...) El tamaño así expuesto es una característica de los miembros en su estatus colectivo como un grupo. En relación con las colectividades, la palabra "grande" significa "consistente de muchos miembros"; tal significado, que define el tamaño por su sola referencia a individuos, no puede aplicarse a los individuos excepto en la medida en que son constitutivos de un grupo (Kramer, 2002, pp. 54-55).

En definitiva, no se requiere otra cosa que individuos para la composición del grupo, pero éste se compone de algo más que de dichos individuos independiente o aisladamente, se compone de individuos en tanto que miembros del grupo en cuestión.

La segunda idea alude a lo que Réaume denominaba derechos sobre bienes participativos. Estos bienes serían un tipo singular de bienes colectivos que se 
caracterizan por acentuar que aquello que merece una valoración positiva es la participación en el proceso de configuración de ese bien colectivo. Estos bienes participativos comparten con la generalidad de los bienes colectivos las notas que generalmente los definen. En primer lugar, son producidos públicamente, esto es, su producción requiere de muchos participantes (no es un requisito necesario, aunque suele producirse en la mayoría de los casos). En segundo lugar, muestran un consumo no rivalizado o, al menos, en niveles de grado de no rivalidad similares a lo que es una característica necesaria de los bienes públicos. (...) Finalmente, muestran un grado limitado excluibilidad, ya que no todos pueden ser excluidos de su disfrute (Réaume, 1988, p. 12). Pero, además de estas características y a diferencia de los restantes bienes colectivos, en los bienes participativos «el carácter público de su propia producción sólo es parte de aquello que es valorado; el bien es la participación» (Réaume, 1988, p. 10). Este tipo de bienes no consisten en algún tipo de producto terminado como la limpieza de las calles, el aire limpio o el alumbrado público, sino que consiste en la participación en la producción de esos bienes públicos. La generación de una sociedad culta; el desarrollo de la amistad (o de la fraternidad o de las actividades lúdicas y deportivas); la práctica de la deliberación pública; o el fortalecimiento de la lengua de uso común quizá puedan ser ejemplos de este tipo de bienes participativos (Réaume, 1994, p. 120). La conclusión no es que estos bienes participativos son bienes que disfrutan los individuos o no, sino que lo central es que son bienes que no pueden ser individualmente disfrutados (Réaume, 1988, p. 11). Por esta razón, son bienes públicos reforzados. Su disfrute no es excluible por razones técnicas (por ejemplo, el alumbrado público) o por razones económicas (una tasa sobre el oxígeno) como en los bienes colectivos en general, sino que la participación es constitutiva del mismo bien, por lo que es una característica esencial que no exista exclusión en su elaboración.

De esta manera, el foco del análisis devendría no una cuestión sobre cómo articular un reduccionismo fuerte o un reduccionismo débil en la articulación de derechos individuales y colectivos, sino en evaluar la plausibilidad moral de justificar la imposición de cargas o deberes correlativos a la satisfacción y garantía de esos bienes participativos. Dicho de otra manera, siguiendo la idea apuntada por el propio Caracciolo de que un derecho se corresponde a un interés suficientemente relevante como para justificar la imposición de deberes, el tema en cuestión es evaluar la relevancia moral de esa protección de bienes de carácter participativo. En la medida en que tal empresa fuese exitosa, se abriría un cauce autónomo para la promoción de derechos colectivos diferenciados de los derechos individuales sin recurrir a tal efecto a fórmulas comunitaristas $u$ ontologías holistas.

Sin embargo, esta no es la senda que sigue Caracciolo. Su análisis se centra en exponer las condiciones de existencia de los derechos colectivos a la identidad cultural descomponiendo desde el punto de vista lógico las siguientes variables: Grupo (G)/Rasgo Cultural (R)/ Derecho colectivo a conservación de Rasgo Cultural (D/R). Así, sus enunciados podrían ser reformulados de la siguiente forma:

1) Todos los Grupos tienen todos los Rasgos Culturales con Derecho colectivo de protección.

2) Todos los Grupos tienen (al menos) un Rasgo Cultural con Derecho colectivo de protección.

3) Existe un único Rasgo Cultural al que todos los Grupos tienen Derecho colectivo de protección.

4) Todos los Rasgos Culturales de un Grupo dado tienen Derecho colectivo de protección.

5) Existe al menos un Grupo con todos los Rasgos Culturales con Derecho colectivo de protección. 
6) Existe al menos un Grupo con un Rasgo Cultural con Derecho colectivo de protección.

Descartadas (4) por falsa y (5) por imperialista, Caracciolo entiende que (1) y (3) deben ser rechazadas por los defensores de los derechos colectivos a la identidad cultural. (1) por lo que tendría de radical cosificación y por ser probablemente imposible, mientras que (3) por impedir la diferencia.

El debate, por tanto, se centraría entre (2) y (6) y, en lo que me corresponde, diría con Caracciolo que es esta última opción la que sería la relevante.

Sin embargo, y esta es la parte final de su ensayo, Caracciolo adopta una última estrategia de estudio que versa sobre la evaluación moral de los presuntos derechos colectivos a la identidad cultural. La controversia no se refiere a la descripción del reconocimiento social o jurídico de dichos derechos, sino a la evaluación de qué rasgos deben ser moralmente respetados. Esta cuestión resulta especialmente exigente porque, a los ojos de Caracciolo, estos rasgos conllevan necesariamente un conflicto de juicios de valor.

Para abordar esta cuestión, señala el profesor, disponemos de dos aproximaciones metaéticas: una escéptica y una objetivista. La primera es irrelevante porque no ofrece criterios de evaluación intergrupales de los juicios de valor en conflicto. Cada rasgo cultural o conjunto de rasgos culturales resultan así inconmensurables. Por lo que, en definitiva, desde el esceptismo ético no podrían defenderse los derechos colectivos a la identidad cultural.

La segunda es, sin embargo, paradójica. Ofrece criterios evaluatorios de la aceptabilidad de los juicios morales y, por tanto, criterios para evaluar la aceptabilidad moral del rasgo cultural que, en cuestión, define al grupo. Sin embargo, el hecho de que sea el rasgo cultural definitorio del grupo, resulta irrelevante para su evaluación moral, por lo que el grupo resulta irrelevante y, por tanto, el discurso de los derechos colectivos también.

Estas consideraciones en el apartado final del ensayo implican un debate de largo alcance que excede el presente trabajo, aunque quisiera significar algún comentario final. En primer lugar, la opción objetivista resulta paradójica en la medida en que los criterios objetivos que se suministren para la evaluación moral de los rasgos culturales no contengan una opción objetivista de carácter contextualista. Bajo dicha etiqueta se encontrarían aquellas opciones metaéticas que suministrarían criterios objetivos de evaluación de los juicios de valor atinentes a la tradición (comunitarismo), a instancias trascendentales (teológicas) o a voluntades (Estado, partido, soberano, etc.). Todas estas opciones presentan caracteres metaéticos objetivistas y creo que, al menos en algunas de sus versiones, serían proclives a promover el reconocimiento moral de todos o de algunos rasgos morales de todos o algunos grupos. Por tanto, cuando Caracciolo defiende el objetivismo moral, defiende una versión del objetivismo moral que se caracteriza por su univocidad.

En segundo lugar, desde el punto de vista de Caracciolo, probablemente, las falencias de las opciones metaéticas escépticas serían predicables tanto de los derechos colectivos como de los derechos individuales por lo que, en este contexto de debate, resultarían irrelevantes.

En tercer lugar, el planteamiento de la evaluación moral del rasgo cultural adolece de las deficiencias que se derivarían de la cosificación del rasgo en cuestión. En la medida en que el centro de gravedad del contenido de los derechos colectivos de identidad cultural se refiera a procesos, en especial los de generación de bienes participativos, parece difícil someterlos a juicios morales comparativos. El caso paradigmático probablemente venga constituido por la lengua de uso común en un grupo. 
En cuarto lugar, la inexistencia de criterios objetivos que señala el escepticismo no implica la inexistencia de juicios de valor moral. Esta afirmación se proyecta en dos dimensiones. Metaéticamente hablando, dichos juicios morales son incomensurables, tanto en términos colectivos como en términos individuales. Por lo que su virtualidad tiene las mismas proyecciones sobre la formulación de juicios morales sobre rasgos colectivos que en aquellos casos en que los juicios morales tienen carácter puramente individual. Éticamente hablando, el escepticismo metaético no implica nihilismo ético sino el reconocimiento de que los juicios morales se corresponden a sentimientos, visiones del mundo o inclinaciones ideológicas en las que, por demás, los contextos culturales juegan un papel relevante.

En quinto lugar, en la medida que las opciones metaéticas escépticas propician la formulación de juicios morales sin sometimiento a criterios objetivos y, por tanto, no susceptibles de verificación, se acentúa el carácter cultural del propio discurso moral. Éste no queda sometido a los marcos de una determinada cultura y de sus eventuales rasgos definitorios de su identidad, sino que la actividad ética es en sí misma un tipo de proceso cultural que, por definición, no queda sometida o limitada por los resultados de las manifestaciones que en cada momento se vayan verificando.

Finalmente, recurro a la vieja opción kelseniana (1991, pp. 42 y ss) de promover una estrategia pragmática de un modus vivendi que se incline por la preferencia práctica de que la tolerancia pueda ser promisoria en la formulación de los juicios de valor sobre los rasgos culturales de los grupos y tanto para inclinarse a su respeto como para ofrecer siempre una puerta de salida a aquellos que quieran abandonar esos rasgos culturales.

En conclusión, el trabajo de Caracciolo se presenta como un esfuerzo típicamente analítico de carácter conceptual sobre un componente conflictivo y objeto de tenso debate de la teoría jurídica contemporánea. Sin embargo, una atenta mirada sobre el análisis desarrollado por el profesor argentino ofrece la conclusión de que el mismo dista de ser puramente conceptual e implica necesariamente la adopción de perspectivas morales en su conformación. Creo que no se ha de entender como un fracaso específico de la tentativa de estudio desarrollada, sino como una manifestación más de que la labor teórica como puramente conceptual encuentra sus límites en las opciones morales y, más allá, ideológicas que sirven de soporte a la construcción conceptual desarrollada.

3. Propuestas de justificación de los derechos colectivos sobre el valor del colectivismo de Miodrag A. Jovanović

Jovanović ha estudiado detenidamente la cuestión de los derechos colectivos y comienza su monografía Collective Rights. A Legal Theory planteando explícitamente que «este no es un tratado en filosofía moral o política y, por consiguiente, no necesita discutir la cuestión del valor de los grupos independientemente de las normas jurídicas existentes» (2012, p. 44). Su aproximación pretende corresponderse con la de un teórico del Derecho que intenta reconstruir con un grado más elevado de abstracción y generalidad los materiales normativos disponibles, tanto internacionales como constitucionales, de los que da larga cuenta. "Una de las características centrales de los actuales meta-teóricos debates metodológicos es que se centran fundamentalmente sobre los conceptos jurídicos más abstractos, tal que el concepto de Derecho» (2012, p. 18), señala, aludiendo a ejemplos como la norma básica o la imputación de Kelsen o las reglas primarias o secundarias en Hart. Sin embargo, en otros muchos casos, la «línea de demarcación entre cómo la teoría del Derecho estudia el Derecho y cómo la práctica jurídica se relaciona con el Derecho deviene progresivamente difusa, ya que nos movemos hacia un nivel de abstracción más bajo 
donde nos enfrentamos a conceptos jurídicos más operativos» (2012, p. 19). El caso de la «personalidad jurídica» es uno de ellos -tanto en Kelsen como en Hart resultan polémicos-, y «todo el follón con los "derechos colectivos" radica exactamente en si el estatus de una personalidad jurídica separada puede extenderse a grupos en tanto que grupos. Esta es una tarea con la que no pueden comprometerse ni las leyes ni los jueces. Entra en el terreno de la teoría jurídica y también requiere de un método de justificación que no es sólo teorético sino también de naturaleza normativo-moral» (2012, p. 44). En definitiva, Jovanović intenta desarrollar un análisis de la normativa existente, tanto jurídico internacional como estatal, que trascienda su singularidad mediante un proceso de abstracción abierto a compontes extrajurídicos.

Esta apertura hacia componentes normativo-morales en el análisis de los derechos colectivos remite a dicotomías ontológicas y axiológicas. Las primeras marcan la disyuntiva entre el individualismo ontológico caracterizado por sostener que, en términos fenomenológicos, todos los grupos son reducibles a sus miembros (según lo que expusimos, esta tesis sería suscrita por Caracciolo) y el colectivismo ontológico que mantiene cierta similitud entre la posición de los individuos y el de algunas entidades colectivas que existirían, en algún sentido, independientemente de sus miembros individuales. Las dicotomías axiológicas remiten al valor individualismo y al valor colectivismo. El primero considera -salvo en las interpretaciones libertariasque «las vidas de los seres humanos individuales tienen un valor último y las entidades colectivas derivan su valor de su contribución a las vidas de los seres humanos individuales» (2012, p. 46). El segundo sostiene, sin embargo, que «una entidad colectiva puede tener valor independientemente de su contribución al bienestar de los seres humanos individuales» (2012, p. 46). Jovanović adhiere a ambos segundos pares. Respecto del primero, siguiendo a Heinze, aborda las condiciones que limitan la eventual arbitrariedad de la autoridad normativa al constituir estas entidades colectivas (2012, pp. 56-65). Respecto del segundo, sostiene su preferencia porque «puede proveer un sustrato más coherente de ciertas formas de derechos colectivos, especialmente aquellos atribuidos a grupos que no están organizados sobre valores liberales de autonomía individual y tolerancia (por ejemplo, los pueblos indígenas)» (2012, p. 46). Mediante un método recursivo ante los críticos, presenta tres ejemplos para fundamentar esta preferencia. En primer lugar, la idea de genocidio como objeto de la normativa internacional tendente a la protección de un bien diferente al de la vida de los individuos adscritos al grupo de que se trate. Esta idea se hace más patente aún en la de etnocidio, que no requeriría esta última dimensión de atentado individual. En segundo lugar, la idea de colisión entre la preferencia individual de (la mayoría de) los miembros del grupo frente a la del grupo como tal. El ejemplo hipotético que propone es una eventual política pública que alternativamente favorezca la comercialización de los productos agrícolas de una comunidad étnica o la promoción cultural museística de su arte tradicional. Probablemente, el interés individual de los miembros del grupo vaya en favor de aquella mientras que el interés colectivo prefiera esta última. En tercer lugar, que el valor individualista presupone, siguiendo a Bobbio, la noción histórica de un tipo de comunidad política que no puede legítimamente ser absolutizada, el valor individualista es sólo una variable contingente de valor colectivista (2012, pp. 54-55).

A partir de estas bases, el profesor de la Universidad de Belgrado desarrolla dos estrategias de justificación que se presentan de largo aliento. La primera se refiere al análisis de las teorías que sustentan la titularidad de derechos en sus diversas facetas. El valor colectivista se proyecta en la necesidad de reconocer la preferencia lexicográfica de la existencia de los entes colectivos sobre la posibilidad de atribuirles derechos que protejan bienes públicos, colectivos y, en especial, participativos que van más allá de las propuestas de Raz (1986), Margalit y Raz (1995), Réaume (1988) o Marmor (2001). En este sentido, Jovanović sostiene una lectura del Raz menos individualista (1995 y 2003) que se acerca al corporativismo de Jones (1999a, 1999b 
y 2000) y a los bienes socialmente irreductibles de Taylor (1995, pp. 137 y ss) y concluye en la razonabilidad fundamentadora del valor colectivista y su superioridad heurística para dar sentido a las prácticas jurídicas nacionales e internacionales sobre la materia (2012, pp. 88-109 y pp. 140-152). La segunda estrategia consiste en argumentar en favor de la categoría de derechos colectivos como un concepto jurídico autónomo y específico frente a los derechos individuales ejercidos colectivamente. Jovanović destaca que la vinculación entre ambas ideas goza de amplio predicamento (por todos: Réaume, 1994; Morauta; Jones, 1999a; Buchanan, 1993). A tal fin, parte de un análisis comparativo entre los derechos individuales y los derechos de grupo. Aquellos pueden ser ejercidos por su titular en lo que constituye la más común de las circunstancias; por un representante del mismo en los casos de incapaces o menores o de manera conjunta por los titulares en los casos de los derechos de reunión, asociación o huelga. Por su parte, los derechos de grupo también pueden ser ejercidos de tres formas. En primer lugar, de forma no individual, a través de un procedimiento colectivo (por ejemplo, un proceso mayoritario de toma de decisiones como en la autodeterminación). En segundo lugar, por medio de un agente o agentes que lo ejercen para el grupo (por ejemplo, quien representa al grupo ante las autoridades educativas con el objeto de fijar el currículum escolar singular de los miembros del mismo). En tercer lugar, en forma de posición dual que puede ser ejercido por cualquier miembro del grupo (por ejemplo, un indígena que pesca en las zonas de especial protección atribuidas a su pueblo) o por un mecanismo colectivo o por un agente (2012, pp. 110-117). La equivalencia entre ellos no es plena. La diferencia más relevante radica en que el titular individual ejerce su derecho en su propio interés, mientras que el ejercicio del derecho por el titular del derecho de grupo lo ejerce en interés de cualquier miembro del grupo.

La segunda alternativa analítica que ensaya Jovanović se refiere al análisis específico de los derechos de grupo. Siguiendo a Mitnick y, en especial, a Baubök (1999, pp. 134 y ss) y Pogge (1997, pp. 191 y ss), clasifica a estos derechos en tres tipos diferentes: derechos de grupo en sí, derechos como grupo específico y derechos de grupo estadístico. Ejemplo de los primeros serían el derecho de los ciudadanos de Oregón de nombrar dos senadores en el Senado federal. Ejemplo de los segundos sería el derecho de los Sijs a montar en bicicleta sin casco. Ejemplo de los terceros sería una representación mínima de los miembros de un grupo racializado o un género en las admisiones universitarias (2012, pp. 121 y 122). Sin embargo, resulta igualmente un intento fallido. La última de las categorías se refiere a acciones afirmativas y no se comparece con el concepto de derecho de grupo. Estas medidas son vistas como excepciones, tienen carácter temporal y dependen de las circunstancias. Ninguna de estas notas sería predicable de los derechos reconocidos a un grupo. La primera de ellas adolecería de una legitimidad especial porque su creación es una variable dependiente de las normas jurídicas que rijan la materia en cuestión. Por decirlo simplificadamente respecto del ejemplo, no hay ningún grupo diferente de aquel que es meramente creado por la ley electoral de Oregón (2012, p. 123 y 132).

Desde estas premisas, la estrategia argumentativa seguida por Jovanović se dirige a identificar cuáles serían los argumentos que permiten identificar un derecho de grupo como un derecho colectivo. Esta distinción entre clases de sujetos (tales como mujeres, minorías sexuales, discapacitados) y colectivos resulta crucial para la definición de los derechos colectivos. De la mano de Miller y su nacionalismo liberal, entre otros, sostiene que han de ser colectividades que tengan un carácter sociológico prelegal y que, por tanto, no sean reducibles a su creación normativa. A este carácter previo se le ha de añadir que detenten unos intereses colectivos propios en cuanto qua grupo que instauren un valor inherente al mismo. Este valor es el presupuesto normativo moral del valor colectivista (2012, p. 131). Éste cualifica, en primer lugar, grupos étnicos y raciales que incluyen a aquellos basados en el color, la ascendencia 
o su carácter nacional; en segundo lugar, grupos religiosos y, en tercer lugar, grupos lingüísticos y culturales. Por el contrario, no se aplicaría a grupos caracterizados por el género, la orientación sexual, el origen social, las condiciones económicas o las opiniones políticas. En definitiva, los grupos que dispondrían de derechos colectivos debieran ser «claros, permanentes, estables, compartiendo un origen común o teniendo características en común; la membresía en ellos debiera no ser opcional, sino inevitable, podrían ser identificados mediante criterios objetivos» (2012, p. 134).

Sobre esta base, el último apartado que Jovanović aborda en su estudio es el relativo a la relación que los derechos colectivos, definidos por referencia al valor colectivista, mantienen con los derechos individuales. Desde Kymlicka, parece un lugar común entre el liberalismo culturalista -Ignatieff, Marmor, Baubök, Buchanan, etc.- la admisión algunas restricciones a los derechos individuales en razón de la protección de derechos de grupo, siempre que se refieran a los derechos de individuos del grupo mayoritario, operando estas limitaciones como protecciones externas de los derechos de los titulares del derecho de grupo de que se trate. Por el contrario, y en expresión de lo que Jovanović etiqueta como valor individualista, dichas restricciones no pueden operar en relación con los derechos individuales de los miembros del grupo en cuestión (inadmisibilidad de las restricciones internas) (Kymlicka,1996, pp. 57-76 y pp. 211-214). Sin embargo, Jovanović analiza pormenorizadamente las modulaciones que esta teoría ha ido desarrollando. El propio Kymlicka reconocía que la propiedad común de la tierra para los pueblos indígenas era un elemento central en la defensa de la identidad del grupo y ello, obviamente, suponía severas restricciones en los derechos individuales de los miembros de dicho colectivo. Más en general, procedía a distinguir entre la violación de libertades civiles básicas -que es inaceptable- y las restricciones a libertades económicas -que pueden ser aceptables. Esta tesis, a los ojos de Jovanović, supone un cambio categorial en esta postura, acercándose, de forma definitiva, a las tesis taylorianas vinculadas al valor colectivista que diferenciaría entre estrictas e innegociables libertades fundamentales y flexibles y adecuables privilegios e inmunidades (2012, pp. 140-150).

Con todo, esta distinción está lejos de adquirir una forma acabada y estable y resulta dificultoso poder delimitar de forma definitiva ambas categorías. A la luz de los trabajos de Rosenfeld en los Estados Unidos y Poulter en el Reino Unido, Jovanović defiende el uso de una multiplicidad de técnicas interpretativas que permitan valorar la adecuación, caso por caso, de la preferencia por el derecho individual o colectivo en conflicto. En sus propios términos: «En el nivel abstracto, esta teoría no puede ofrecer un criterio concluyente para delinear la esfera de los derechos individuales que han de ser tratados como inviolables. Lo más que puede hacer es indicar algunas propuestas teóricas, así como establecer prácticas internacionales y locales de derechos humanos que sean relevantes para la discusión» (2012, p. 151). Entre ellas, ocupa un lugar preferente el denominado test de proporcionalidad, «de acuerdo con el cual las restricciones a los derechos y libertades individuales son legítimas solo si un Estado puede probar que tiene un interés concluyente y que la realización de tal interés no podría ser conseguido mediante otras medidas menos restrictivas» (2012, p.152). En conclusión, Jovanović sostiene que «esta teoría jurídica de los derechos colectivos basada en el punto de vista normativo-moral del valor del colectivismo abre la puerta a la posibilidad de que los derechos colectivos se antepongan no solo a los derechos individuales de los no miembros, sino también a los de los miembros del grupo» (2012, p. 151).

El último aspecto a destacar del trabajo del profesor de la Universidad de Belgrado se refiere a la posibilidad de que estos derechos colectivos defendidos en su teoría sean susceptibles de ser valorados como universales. Su respuesta es afirmativa (2012, p. 182). Siguiendo la evolución de diversas regulaciones jurídicas 
de carácter fundamentalmente internacional, concluye con Crawford ${ }^{4}$ (1988, pp. 57 y ss) en que hay dos categorías de derechos colectivos universales: los relativos a la existencia de los pueblos (autodeterminación, derechos de las minorías, derecho a la existencia física) y los relativos a la coexistencia con otros pueblos (derecho a la paz y seguridad internacional, permanente soberanía sobre sus recursos naturales, al desarrollo y al medio ambiente). A diferencia de algunos derechos individuales cuya universalidad pudiera estar, a su juicio, bajo controversia en el panorama internacional, los derechos colectivos universales, en concreto, los derechos de los pueblos a la autodeterminación, de las minorías a su preservación física y cultural y de los pueblos indígenas a la propiedad de su tierra, se encuentran justificados en numerosas culturas y por doctrinas comprensivas, incluyendo el individualismo occidental (2012, p. 190). Las dificultades que se plantean a los mismos «no [representan] obstáculos insuperables para aquellos que defienden la sostenibilidad de los derechos colectivos universales. Tal y como vimos, esta construcción conceptual puede estar, a la vez, fundamentada filosóficamente y sostenida por elementos de la práctica jurídica internacional» (2012, p. 195).

En conclusión, Jovanović ha tratado de conformar una teoría general de los derechos colectivos articulando reflexión filosófico moral y política con reformulación abstracta de los materiales normativos generados en la materia en los últimos cincuenta años. Las notas centrales de la misma son la atribución de la titularidad y ejercicio de dichos derechos a un tipo de colectivos singulares vinculados a la idea de pueblo, minoría o comunidad religiosa o lingüística; el reconocimiento de un interés propio del grupo no reconducible a los intereses individuales de sus integrantes; la eventual potencia de estos derechos para poder imponerse, según las circunstancias, a los derechos individuales de los miembros del grupo $-\mathrm{y}$, obviamente, también a los de no miembros-, y su carácter universal. El fundamento último de estas características se basa en la defensa del valor colectivista en su contraposición con el tradicional valor individualista de la cultura occidental de los derechos individuales.

En las líneas que siguen quisiera apuntar algunas cuestiones que este planteamiento ha dejado, según mi valoración, con insuficiente justificación. En primer lugar, la metodología empleada adolece de una ambivalencia inconcluyente. Una aproximación teórico jurídica -en especial de corte positivista- implica una reconstrucción conceptual de los materiales jurídico normativos disponibles, así como de la revisión de las construcciones dogmáticas que dan cuenta de ellos. Sin embargo, el debate filosófico subyacente sobre la aceptabilidad moral o política de sus contenidos no se corresponde con dicha metodología. Las alternativas se decantarían por una teoría jurídica de corte postpositivista o por el mantenimiento de dos debates paralelos, simétricos, pero intangentes. En segundo lugar, requiere una especial atención la variabilidad histórica de la valoración de los criterios de identificación de los grupos y, por tanto, de la ontología que puedan presentar. Pongo dos ejemplos que, a falta de exactitud, pueden aportar claridad. Probablemente, en el establecimiento de fronteras entre los Países Bajos y Bélgica en el siglo XIX, la religión constituyó el criterio decisivo por el que los flamencos, de mayoría católica, se integraran en Bélgica. Hoy en día, el predominio del factor lingüístico supondría, probablemente, la consecuencia contraria. El segundo ejemplo aludiría a la significación de la categoría de trabajador o, más tradicionalmente, de proletario. Las condiciones socio-económicas parece que no computan en la conformación de grupo con derechos colectivos y, sin embargo, cabe sostener la relevancia identitaria de la clase -cuando no de la casta- y, sobre todo, la utilidad heurística que proporciona

\footnotetext{
4 Crawford en su trabajo de 1988 realiza un minucioso seguimiento de la normativa internacional relevante desde los años 70 y centra su atención en el Convenio 169 de la OIT que entró en vigor al año siguiente, y en el proyecto de Declaración de Naciones Unidas sobre Pueblos Indígenas y Tribales que finalmente se aprobó el 1 de septiembre de 2007.
} 
para concebir el derecho de huelga como de titularidad típicamente colectiva y de ejercicio individual (frente al derecho al trabajo que sería individual del trabajador y de ejercicio, en la mayoría de los casos, de carácter colectivo).

La tercera consideración aludiría a la entidad sustantiva del valor colectivista. Sospecho que el autor ha evitado conscientemente el uso del término comunitarista, de larga referencia en este contexto. Más allá de los términos, los autores que frecuentemente vienen en su auxilio, con Taylor en lugar primordial, parecen consolidar esta valoración de continuidad. Si este es el caso, la primera cuestión se referiría a la exposición de las razones por las que se ha seguido este discreto silencio y que resultarían interesantes para examinar la coherencia metodológica de la teoría. La segunda haría referencia, en relación con la primera observación arriba indicada, a cómo compatibilizar las pretensiones generalistas de la teoría jurídica a las que se adhiere el autor con tesis morales característicamente particularistas. Es cierto que ambas reflexiones versan sobre objetos de estudio diferenciados, pero observo problemas de encaje. Las aproximaciones genéricamente postpositivistas pueden concurrir tanto con discursos universalistas (paradigmáticamente, el neoconstitucionalismo garantista) como particularistas (por caso, algunas versiones dworkinianas), pero la pretensión de una aproximación teórico jurídica positivista y de enfoque generalista sólo podría articularse sosteniendo presupuestos escépticos en moral (a lo Kelsen) u optando por una aproximación ética de corte universalista (a lo Hart). La construcción de una teoría general de los derechos colectivos de impronta jurídico-positivista y generalista sobre bases comunitaristas en materia moral requiere aún de mayores justificaciones. Es cierto que una parte importante de la estrategia analítica seguida por Jovanović alude al estudio de los materiales normativos y dogmáticos sobre la materia. Sin embargo, construir una teoría general sobre la base de una opción moral contextualista parece complejo. Finalmente, la contraposición entre valor individualista y valor colectivista como criterio de diferenciación entre una teoría de los derechos colectivos frente a una de los derechos de grupo supone una dicotomía discutible. Quizá la referencia a los trabajos de Seymour (2008) y Sauca (2014) puede aportar algunas luces sobre cómo defender la idea de derechos colectivos sin suscribir el comunitarismo.

\section{Consideraciones finales}

La obra de Miodrag A. Jovanović comparte con Ricardo Caracciolo un mismo aire de familia en sus parámetros generales sobre la teoría jurídica. Destacaría como comunes sus encuadramientos en la tradición de lo que solía llamarse el iuspositivismo metodológico; una pretensión generalista en el enfoque de la determinación del concepto de Derecho y un método de estudio volcado en el análisis del lenguaje común usado por los juristas. Kelsen, Hart, MacCormick, Raz serían autores que nutren centralmente sus respectivas fuentes de inspiración. Entre las diferencias, además de las contextuales, señalaría una mayor relevancia de la lógica para Caracciolo, mientras que Jovanović presenta una apertura más explícita hacia cuestiones morales, políticas y sociales; una proclividad a la receptividad del Derecho internacional para el profesor de la Universidad de Belgrado, mientras que el marco conceptual del profesor de la Universidad de Córdoba es genéricamente el estatal, y finalmente, asunto no irrelevante, que la cuestión de los derechos colectivos resulta colateral para el argentino y central para el serbio.

Sin embargo, el punto más importante que pretendo destacar en la comparativa entre ambos autores es cómo un común background teórico, calificable como iuspositivista metodológico, es presentado como soporte de conclusiones teóricamente incompatibles en perspectiva moral y política. Ambos autores comparten explícitamente su adscripción a una tradición teórico jurídica que responde a las notas de pretensión de generalidad sobre los concretos sistemas jurídicos analizados, 
autonomía de lo jurídico en cuanto a sus métodos y exigencia de objetividad en la determinación de los conceptos jurídicos independientemente de opciones morales y políticas subjetivamente profesadas. Pues bien, el resultado es que tanto Caracciolo como Jovanović hacen seguir sus conclusiones teórico jurídicas de sus consideraciones político-morales. Caracciolo concluye en la inadmisibilidad de la categoría de derechos colectivos mediante un análisis conceptual de los mismos, pero sobre un fundamento político-moral que podría etiquetar de carácter liberal universalista. Jovanović alcanza la conclusión opuesta: los derechos colectivos constituyen una categoría jurídica plausible, basada en una filosofía colectivista. La conclusión no es que ambos operan en transgresión de los presupuestos metodológicos que dicen profesar en su teoría del Derecho, sino que son un buen ejemplo de cómo ésta no puede seguir sosteniendo su presunta asepsia ideológica.

\section{Bibliografía}

Bengoetxea, J. (2015). El derecho a decidir. Un planteamiento desde la teoría del Derecho internacional. lura Vasconiae, 12, pp. 339-361.

Barry, B. (2001). Culture and Equality. An Egalitarian Critique of Multiculturalism. Cambridge: Cambridge University Press.

Collective Rights (1991). Canadian Journal of Law \& Jurisprudence, 4(2), 217-421

Baubök, R. (1999). Liberal Justification for Ethnic Group Rights. En Ch. Joppke y S. Lukes, Multicultural Questions (pp. 133-157), Oxford: Oxford University Press.

Buchanan, A. (1993). The Role of Collective Rights in the Theory of Indigenous Peoples' Rights. Transnational Law \& Contemporary Problems, 1(3), pp. 89-108.

Buchanan, A. (1994). Liberalism and Group Rights. En J. Coleman y A. Buchanan (eds.), In Harm's Way - Essays in Honour of Joel Feinberg (pp. 1-15), Cambridge: Cambridge University Press.

Caracciolo, R. (1991). Derecho y decisiones colectivas. Revista del Centro de Estudios Constitucionales, 10, Septiembre-Diciembre, pp. 25-48.

Caracciolo, R. (1994). La noción de sistema en la teoría del Derecho. México: Fontamara.

Caracciolo, R. (2001). Realismo moral vs. positivismo jurídico. P. Comanducci e R. Guastini (dir.), Analisi e diritto (pp. 37-44). Torino: Giapichelli editore.

Caracciolo, R. (2002). ¿Existen los derechos colectivos? En S. Pozzolo (a cura di), La legge e i diritti (pp. 267-273), Torino: Giapichelli.

Caracciolo, R. (2006). Acción colectiva y justicia. Derecho y Humanidades, 12, pp. 95102.

Caracciolo, R. (2009). ¿Existen los derechos colectivos?. En El Derecho desde la Filosofía. Ensayos (pp. 261-267), Madrid: Centro de Estudios Políticos y Constitucionales.

Crawford, J. (1988). The Rights of Peoples: "Peoples" or "Governments"? En J. Crawford (ed.), The Rights of Peoples (pp. 7-68), Oxford: Oxford University Press.

Cruz Parcero, J.A. (1998). Sobre el concepto de derechos colectivos. Revista Internacional de Filosofía Política, 12, pp. 95-115.

Cruz Parcero, J.A. (2007). El lenguaje de los derechos. Ensayo para una teoría estructural de los derechos. Madrid: Trotta.

García Inda, A. (2001). Materiales para una reflexión sobre los derechos humanos colectivos. Madrid: Instituto de Derechos Humanos Bartolomé de las Casas, Dykinson.

González Amuchastegui, J. (2004). Autonomía, dignidad y ciudadanía. Valencia: Tirant lo Blanch.

Jones, P. (1999a). Group Rights and Group Oppression. Journal of Political Philosophy, 4(7), pp. 353-377.

Jones, P. (1999b). Human Rights, Group Rights and People's Rights. Human Rights Quarterly, 1(21), pp. 80-107.

Jones, P. (2000). Individuals. Communities and Human Rights. Review of International Studies, 26, pp. 119-215.

Jovanović, M.A., (2012). Collective Rights. A Legal Theory. Cambridge: Cambridge 
University Press.

Kelsen, H. (1991). ¿Qué es Justicia?. En ¿Qué es Justicia? (pp. 35-63), Barcelona: Ariel. Kelsen, H. (2008). Teoría General del Estado. México: Coyoacán.

Kramer, M.H. (2002). Rights without Trimmings. En M.H. Kramer, N.E. Simmonds y H. Steiner, A Debate over Rights. Philosophical Inquiries (pp. 7-102), New York: Oxford University Press.

Kramer, M.H. (2017). Liberalism with Excellence. Oxford: Oxford University Press.

Kymlicka, W. (1996). Ciudadanía multicultural. Barcelona: Paidós.

López Calera, N. (2000). ¿Hay derechos colectivos? Individualidad y socialidad en la teoría de los derechos. Barcelona: Ariel.

López Calera, N. (1992). Yo, el Estado. Bases para una teoría sustancializadora (no sustancialista) del Estado. Madrid: Trotta.

MacCormick, N. (1977). Rights in legislation. En P.M.S. Hacker y J. Raz (eds.), Law, Morality, and Society. Essays in Honour of H.L.A Hart (pp. 189-209), Oxford: Clarendon Press.

MacCormick, N. (1982). Children rights: A test-Case. En Legal Right and Social Democracy (pp. 154-166), Oxford: Clarendon Press.

Margalit, A. y Raz, J. (1995). National Self-Determination. En W. Kymlicka (ed.), The Rights of Minority Cultures (pp. 79-92), Oxford: Oxford University Press.

Marmor, A. (2001). De We Have a Right to Common Goods?. Canadian Journal of Law and Jurisprudence, 2(14), pp. 213-225.

Pogge, T. (1997). Group Rights and Ethnicity. En I. Shapiro y W. Kymlicka (eds.), Ethnicity and Group Rights (pp. 187-221), New York \& London: New York University Press.

Réaume, D. (1988). Individuals, Groups, and Rights to Public Goods. The University of Toronto Law Journal, 38 (1) (Winter), pp. 1-27.

Réaume, D., (1994). The Group Right to Linguistic Security: Whose Right, What Duties?. En J. Baker (ed.), Group Rights (pp.118-141), Toronto: University of Toronto Press.

Raz, J. (1986). Morality of Freedom. Oxford: Oxford University Press.

Raz, J. (1994). Multiculturalism. A Liberal Perspective. Dissent, 41 (Winter), pp. 67-79.

Raz, J. (1995). Rights and Polítics. Indiana Law Journal, 1(71), pp. 27.44.

Raz, J. (1998), «Multiculturalism», en Ratio luris, vol. 11, n. 3, pp. 193 - 205.

Raz, J. (2003). Comments and Responses. En L.H. Meyer, S. Paulson y T. Pogge (eds.), Rights, Culture, and the Law-Themes from the Legal and Political Philosophy of Joseph Raz (pp. 253-274), Oxford: Oxford University Press.

Rodríguez Abascal, L. (2000). Las fronteras del nacionalismo. Madrid: Centro de Estudios Políticos y Constitucionales.

Rodríguez Abascal, L. (2003). On the admissibility of group rights. Annual Survey of International \& Comparative Law, 9(1), pp. 101-110.

Sauca, J.M. (2010). Identidad y Derecho. Nuevas perspectivas para viejos debates. Valencia: Tirant lo Blanch.

Sauca, J.M. (2014). The Canadian School of Diversity's New Influences on the Theory of Collective Rights in Spain. A Critical Review of Seymour's Contribution. En A.G. Gagnon y J.M. Sauca (eds.), Negotiating Diversity. Identity, Pluralism and Democracy (pp. 61-86), Bruxelles: PIE Peter Lange.

Seymour, M. (2008). De la tolérance à la reconnaissance. Une théorie libérale des droits collectifs. Montréal: Boréal.

Taylor, Ch. (1995). Philosophical Arguments. Cambridge, Mass: Harvard University Press.

Torbisco Casals, N. (2006). Group Rights as Human Rights. A liberal Approach to Multiculturalism. Dordrecht: Springer.

Van Dyke, V. (1974). Human Rights and the Rights of Groups. American Journal of Political Science, 18, pp. 725-741.

Van Dyke, V. (1980). The Cultural Rights of Peoples. Universal Human Rights, 2(2) (AprJun), pp. 1- 21. 